\title{
Rejuvenation of bulges by bars: evidence from stellar population analysis
}

\author{
Dimitri A. Gadotti ${ }^{1}$ and Paula Coelho ${ }^{2}$ \\ ${ }^{1}$ European Southern Observatory \\ email: dgadotti@eso.org \\ ${ }^{2}$ Universidade Cruzeiro do Sul \\ email: paula.coelho@cruzeirodosul.edu.br
}

\begin{abstract}
We obtained stellar ages and metallicities via spectrum fitting for a sample of 575 bulges with spectra available from the SDSS. Using the whole sample, where galaxy stellar mass distributions for barred and unbarred galaxies are similar, we find that the distribution of bulge ages in barred galaxies shows an excess of populations younger than 4 Gyr, when compared to bulges in unbarred galaxies. KS statistics confirm that the age distributions are different with a significance of $99.94 \%$. If we select sub-samples for which the bulge stellar mass distributions are similar for barred and unbarred galaxies, this excess vanishes for galaxies with low-mass bulges, while for more massive bulges we find a bimodal stellar age distribution for barred galaxies only, corresponding to two normal distributions with mean ages of 10.4 and 4.7 Gyr (see Fig. 1). These results lend strong support to models in which bars trigger star formation activity in the centers of galaxies. We also find twice as much AGN among barred galaxies, as compared to unbarred galaxies, for low-mass bulges. Full results are in Coelho \& Gadotti (2011).
\end{abstract}

Keywords. galaxies: bulges, galaxies: evolution, galaxies: stellar content

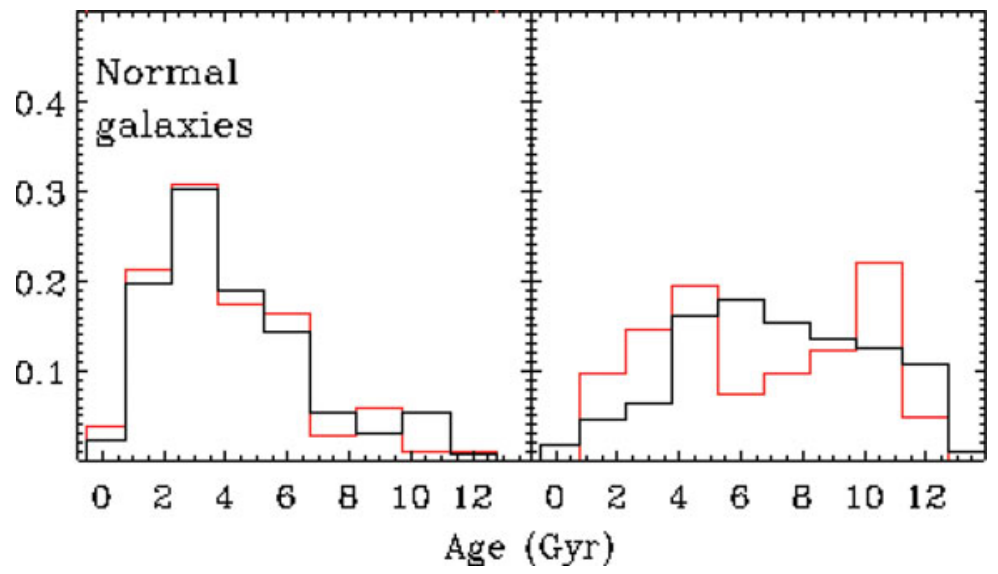

Figure 1. Normalized distributions of the mean stellar ages of bulges in barred and unbarred galaxies. For low mass bulges $\left(<10^{10.1} \mathrm{M}_{\odot}-\right.$ left $)$ the distributions are similar, whereas for high mass bulges $\left(>10^{10.1} \mathrm{M}_{\odot}\right.$ - right) only barred galaxies have a bimodal distribution of bulge mean stellar ages, with a significant young component. Adapted from Coelho \& Gadotti (2011).

\section{Reference}

Coelho, P. \& Gadotti, D. A. 2011, ApJ Letters 743, 13 